\title{
Entry Into New Therapeutic Areas: The Effect of Alliance on Clinical Trials
}

Therapeutic Innovation \& Regulatory Science 2019, Vol. 53(5) 648-653 (C) The Author(s) 2018 Article reuse guidelines: sagepub.com/journals-permissions DOI: 10.1 | $77 / 21684790188$ I 829 tirs.sagepub.com

\author{
Kinya Okada, PhD', and Shintaro Sengoku, $\mathbf{P h D}^{\prime}{ }^{\top}$
}

\begin{abstract}
Background: Entry into a new therapeutic area, that is, one in which a pharmaceutical firm lacks experience, is a considerable challenge for firms that need to overcome scientific and technological barriers. To address this issue, the present study aims to explore the potentiality of alliances in an empirical manner. Methods: From the clinical trials sponsored by 20 major pharmaceutical firms during 2008-2016 listed at ClinicalTrials.gov $(n=14,94 \mid$ clinical trials), cases of entering a new therapeutic area for a pharmaceutical firm were extracted $(n=73)$, followed by statistical analyses to evaluate the effect of alliances in this regard. Results: We found that the average number of participating organizations in the cases of entering a new therapeutic area was significantly larger than that in the cases of entering an area in which firms had experience $(P<.0 \mathrm{I})$, suggesting that alliance has a positive effect on new therapeutic entry for these pharmaceutical firms. Second, we found that the cases of partnering with nonindustrial or nongovernmental organizations (ie, universities, research institutes, hospitals, funding agencies, and others; $\mathrm{n}=32$ of the 73) were significantly associated with these new entry trials (adjusted odds ratio $=1.1, P<.05$ ). Furthermore, we identified that 10 of the 32 clinical trials were associated with rare diseases, which is an overrepresentation compared to the occurrence in the universe $\left(1015\right.$ of the 14,$\left.941 ; P<10^{-5}\right)$. Conclusions: These findings strongly suggest the importance of alliances with diversified partners in new therapeutic entry and also provide a basis for further detailed investigation of key success factors for pharmaceutical firms.
\end{abstract}

\section{Keywords}

pharmaceutical business, entry strategy, open innovation, rare disease, drug repurposing, drug repositioning

\section{Introduction}

Since the late 2000s, pharmaceutical firms have been facing considerable challenges because of the so-called patent cliff, ${ }^{1}$ significantly increasing research and development (R\&D) expenditure, ${ }^{2}$ and decreasing productivity, ${ }^{3}$ which has forced reconfiguration of their $R \& D$ strategy. ${ }^{4}$ One promising approach is a category leadership strategy that narrows down the focus on therapeutic areas and maximizes profitability. ${ }^{5}$

By taking the category leadership strategy, Biogen Inc, for instance, has strengthened its category leadership in the neurologic disease area with a particular focus on multiple sclerosis. ${ }^{6}$ Biogen entered this area with interferon beta-1a for relapseremitting multiple sclerosis and established its leading position with other related products. ${ }^{7}$ At the same time, the firm disposed of or terminated several programs, such as oncology and cardiology, that were out of its category focus. ${ }^{6}$ Conversely, Gilead Sciences, known as a category leader with a suite of antivirus therapies, announced the acquisition of Kite Pharma, Inc, one of the leading developers of chimeric antigen receptor (CAR)-T cell immunotherapies, ${ }^{8}$ aiming to enter a new therapeutic area to build up a new category pillar.

One of the possible difficulties when entering new therapeutic areas is the entry barrier, ${ }^{9}$ which is defined in economic theories of competition as additional costs that must be incurred by entrants, not by incumbents. ${ }^{10}$ In the pharmaceutical industry, the entry barrier into new therapeutic areas is critical from a technological aspect in particular because competitive technologies are highly expertized and specialized to each therapeutic area. For example, the delivery of drugs through the blood-brain barrier is one of the most crucial technologies, especially in the neurologic area. ${ }^{11}$

It is not easy for new entrants to develop new technological capabilities in a target therapeutic area that has yet to be explored. The above-mentioned drastic transformation of a firm's strategy could hardly be achieved through organic and incremental growth. Mergers and acquisitions (M\&A) ${ }^{12}$ and proactive license-in ${ }^{13}$ could be a means of ensuring new entry

\footnotetext{
I School of Environment and Society, Tokyo Institute of Technology, Minato-ku, Tokyo, Japan
}

Submitted 26-Apr-2018; accepted 26-Jul-2018

\section{Corresponding Author:}

Shintaro Sengoku, PhD, School of Environment and Society, Tokyo Institute of Technology, Minato-ku, Rm. 908N Campus Innovation Centre (ClC), 3-3-6 Shibaura, Meguro-ku, Tokyo 108-0023, Japan.

Email: sengoku@mot.titech.ac.jp 
efforts; however, little is known about how such types of alliances affect entries into new therapeutic areas from the technological perspective.

To address this issue, the present study explores the effect of alliance on entries into new therapeutic areas and provides insights on pharmaceutical firms' entry strategies. In practice, performing clinical trials or translational research on humans is the primary step for a pharmaceutical firm to enter a new therapeutic area for both cases of its own and in-licensed drug candidates. Therefore, an empirical approach was adopted based on a widely used clinical trials database.

An alliance refers to an arrangement in which 2 or more organizations share their own resources to achieve their goal while maintaining their own identities. ${ }^{14}$ Among the traditional motives, such as market entry, improved efficiency, and risk reduction, ${ }^{15}$ the present study focuses on those associated with entries into new therapeutic areas. As mentioned above, facing an entry barrier to a target therapeutic area, pharmaceutical firms may be inclined to engage in an alliance to fill gaps with the complementary external capabilities of a partner. Based on these considerations, we tested whether the number of alliances with academic, industry, and governmental players has a positive effect on a pharmaceutical firm's entry into a new therapeutic area.

\section{Methods}

ClinicalTrials.gov is a database developed and maintained by the National Institutes of Health (NIH) and the Food and Drug Administration (FDA) in the United States. ${ }^{16}$ In order to facilitate analysis of the complete set of clinical trials, the data from ClinicalTrials.gov have been reformatted and restructured as a relational database called the Aggregate Analysis of ClinicalTrials.gov (AACT) database by the Clinical Trials Transformation Initiative (CTTI) ${ }^{17}$ In this study, the latest AACT database as of October 2017 was downloaded. Information on clinical trials and collaborations was retrieved from the database. Hereafter, the names of entities and their attributes in the database are written in italic typeface.

Medical Subject Headings (MeSH), created and updated by the National Library of Medicine (NLM), is a comprehensive, hierarchical, and controlled vocabulary for indexing journal articles and books in the life sciences. ${ }^{18}$ It primarily serves as a thesaurus that facilitates searching and is also used to characterize which diseases are studied in clinical trials registered in ClinicalTrials.gov ${ }^{16,17}$; more specifically, clinical trials are associated with disease-related MeSH terms. In this study, the $2017 \mathrm{MeSH}$ thesaurus broadest disease classification level, consisting of 27 therapeutic categories, was used for simplicity, as previously used. ${ }^{19}$

Whether a clinical trial $\mathrm{c}$ represents entry into a new therapeutic area depends on the previous experience of clinical trials in the area. In this study, a clinical trial is called "entry" when the pharmaceutical firm had no other clinical trials in the area for at least 5 years since the last clinical trial in the area at

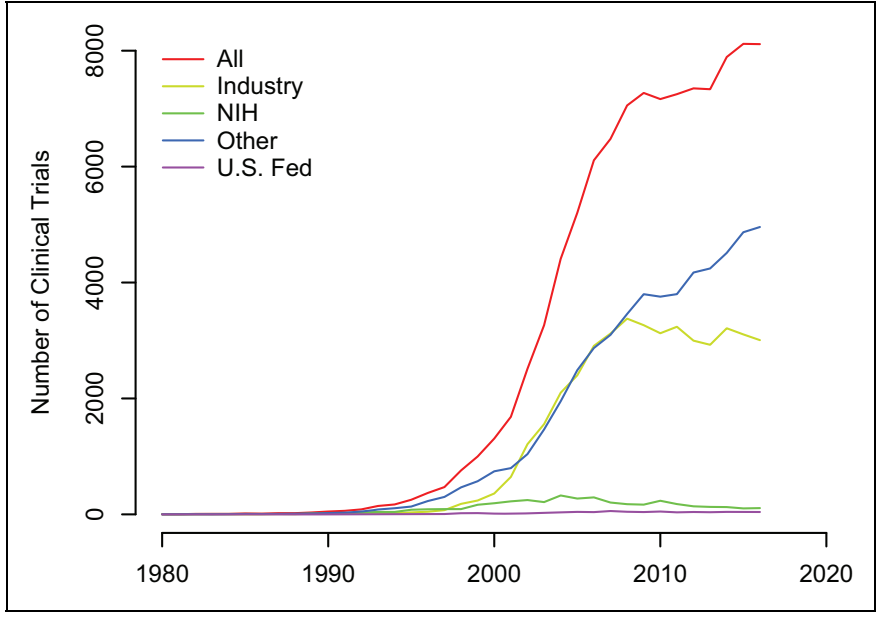

Figure I. Annual number of clinical trials in agency classes. The horizontal $X$-axis indicates year when clinical trials started. The vertical axis indicates how many clinical trials started.

the time of the trial. This interval period was set because the time required to take a product from the start of patient testing in phase II to regulatory approval ranges from 1 to 6 years. ${ }^{20}$ Note, however, that changing it within the range from 4 to 6 years did not affect the conclusion (data not shown).

Let $a_{c}$ be a variable indicating the number of participating organizations in clinical trial c. As the AACT database provides information not only on organizations but also on organization type (industry, NIH, U.S. Fed, or other), $a_{c}$ can be broken down into 4 elements, that is, $x_{1}, x_{2}, x_{3}$, and $x_{4}$, corresponding to the number of organizations of each of these types. In order to evaluate the effects of these types of alliance, a logistic regression model in which 4 elements are linearly combined was used as follows:

$$
\log \left(\frac{P}{1-P}\right)=\beta_{0}+\beta_{1} x_{1}+\beta_{2} x_{2}+\beta_{3} x_{3}+\beta_{4} x_{4}
$$

where $P$ is the probability of a trial being an entry into a new therapeutic area and $\beta$ is a weight vector.

All statistical analyses were performed using the R statistical software (version 3.4.1).

This article does not contain any studies with human or animal subjects performed by any of the authors.

\section{Results}

A schematic representation of data processing flow is shown in Figure S1. First of all, 111,627 drug interventional trials were extracted from the AACT database. These trials were conducted in 188 countries, but mainly in the United States, accounting for $50 \%$. Statistics on the sponsors entity shows that most of the trials were led by other and industry agency classes, accounting for $53 \%$ and $42 \%$, respectively. The annual number of clinical trials led by each agency class also indicates 


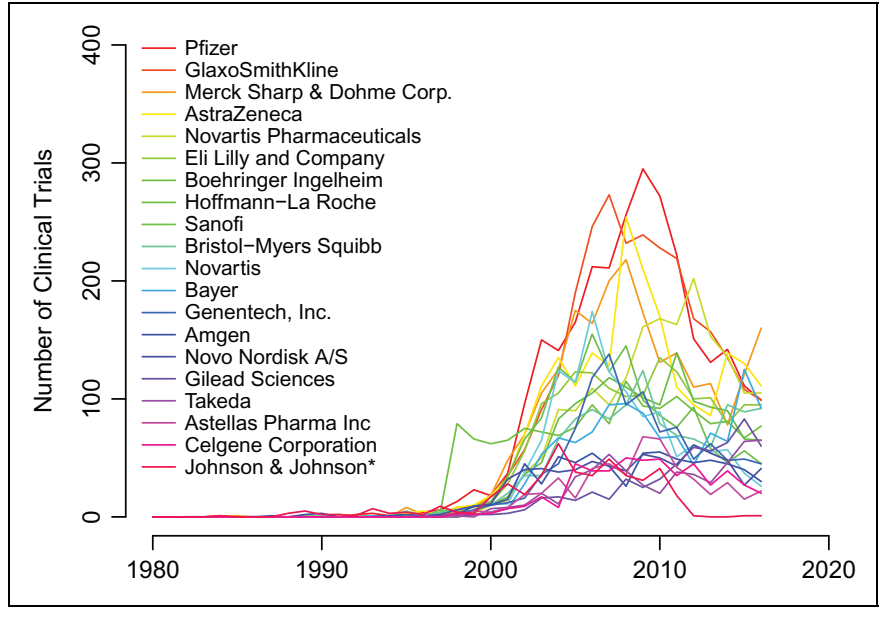

Figure 2. Annual number of clinical trials of top 20 pharmaceutical firms. The horizontal axis indicates year when clinical trials started. The vertical axis indicates how many clinical trials started. *Johnson \& Johnson Pharmaceutical Research \& Development, L.L.C.

that these 2 major classes had dominated since the late 1990s, as shown in Figure 1. In addition, this suggests another intriguing fact; while the annual numbers of clinical trials in cases of both other and industry agency classes had been consistently increasing until the late 2000s, only the trend of the industry agency class turned over in 2008. Since then, the annual number of clinical trials led by the industry agency class has tended to decrease. The cause is not known for certain, but one possible reason is the so-called patent cliff in the late 2000s. ${ }^{1}$ To avoid a possible confounding effect due to this trend shift, this study first focused on the period from 2008 to 2016.

In the database, 58,461 of the drug interventional trials were related to at least 1 organization belonging to the industry agency class; that is, the trials included participation by industry-related organizations as either a lead or a collaborator. In order to set the focus on organizations, the number of clinical trials for each organization belonging to the industry agency class was evaluated, and the top 20 were chosen from 4684 organizations for further analysis. The chosen organizations consisted mainly of global large pharmaceutical firms, as listed in Figure 2. Clinical trials in which at least 1 of these firms participated accounted for approximately half of the industryrelated clinical trials (46\%). Furthermore, the annual number of clinical trials in which they participated tended to decline over the last decade, as did that of the industry agency class overall in Figure 1. Although this does not cover all clinical trials, the clinical trials in which the target organizations participated can therefore be regarded as reasonable representatives of industryrelated clinical trials.

According to the MeSH thesaurus, ${ }^{18} 27$ therapeutic categories were assigned to 14,941 clinical trials in which the top 20 organizations participated from 2008 to 2016 . To overlook distribution of clinical trials among therapeutic areas, a therapeutic area map of clinical trials was drawn for each organization, as shown in Figure S2. As one of the remarkable overall trends among those maps for the 20 organizations, their clinical trials in various therapeutic areas began to be registered in the late 1990s or at the latest in the early 2000s. The map for GlaxoSmithKline Pharmaceuticals Ltd (hereafter referred to as GSK) is shown in Figure 3 as an example, not a representative. The map shows that GSK conducted a clinical trial in the therapeutic area of eye diseases in 2009. Since it had no experience with clinical trials in the area before 2009, the trial can be considered as entry into a new therapeutic area. In the same way, 73 trials in which the target organizations participated were identified as entry into new therapeutic areas.

The number of participating organizations in entry clinical trials was significantly larger than that in nonentry clinical trials $(1.487 \pm 0.987$ for nonentry and $1.822 \pm 1.206$ for entry clinical trials; $t$ test $P<.01$ ). In order to further investigate the effect of agency classes on entry, the number of participating organizations was broken down into the number in each agency class, and then a regression analysis was performed in which those broken factors were linearly combined. The result, as shown in Table 1, is that the number of participating organizations belonging to the other agency class was significantly associated with entry clinical trials (adjusted odds ratio $=1.1$, Wald test $P<.05$ ), indicating that partnering with organizations belonging to the other agency class has a positive effect on entry into new therapeutic areas.

Further investigation was made for 32 of the entry clinical trials in which at least 1 organization belonging to the other agency class participated, as shown in Table S1. First, agencies were classified into the following 5 subgroups: university, research institute, hospital, funding agency, and other; the allocations were 14, 4, 15, 6, and 5, respectively. Second, to test the hypothesis that observed cases work as a cue to direct drugs toward rare diseases, we examined the matching of given MeSH terms of each clinical trial to Orpha.net, a database of approximately 6000 rare diseases. ${ }^{21}$ The result showed that 10 of the 32 clinical trials were associated with rare diseases, which is an overrepresentation compared to the occurrence in the universe $\left(1015\right.$ of 14,$\left.941 ; P<10^{-5}\right)$, supporting that collaboration of firms with academia focusing on rare diseases would be adequate in entry clinical trials. Third, in order to observe whether these interventional compounds were thereafter successfully marketed, the first launch year of each compound was confirmed with the EvaluatePharma database, as shown in Table S1. Interestingly, we found that 25 of the 32 compounds $(78 \%)$ were already on the market at that time of starting the clinical trials, suggesting that pharmaceutical firms were likely to leverage marketed drugs rather than ones under development for entry into new therapeutic areas.

\section{Discussion}

As alliances have been increasingly important in the pharmaceutical industry, various types of partnership models have been proposed. ${ }^{4,22}$ In particular, so-called university- 


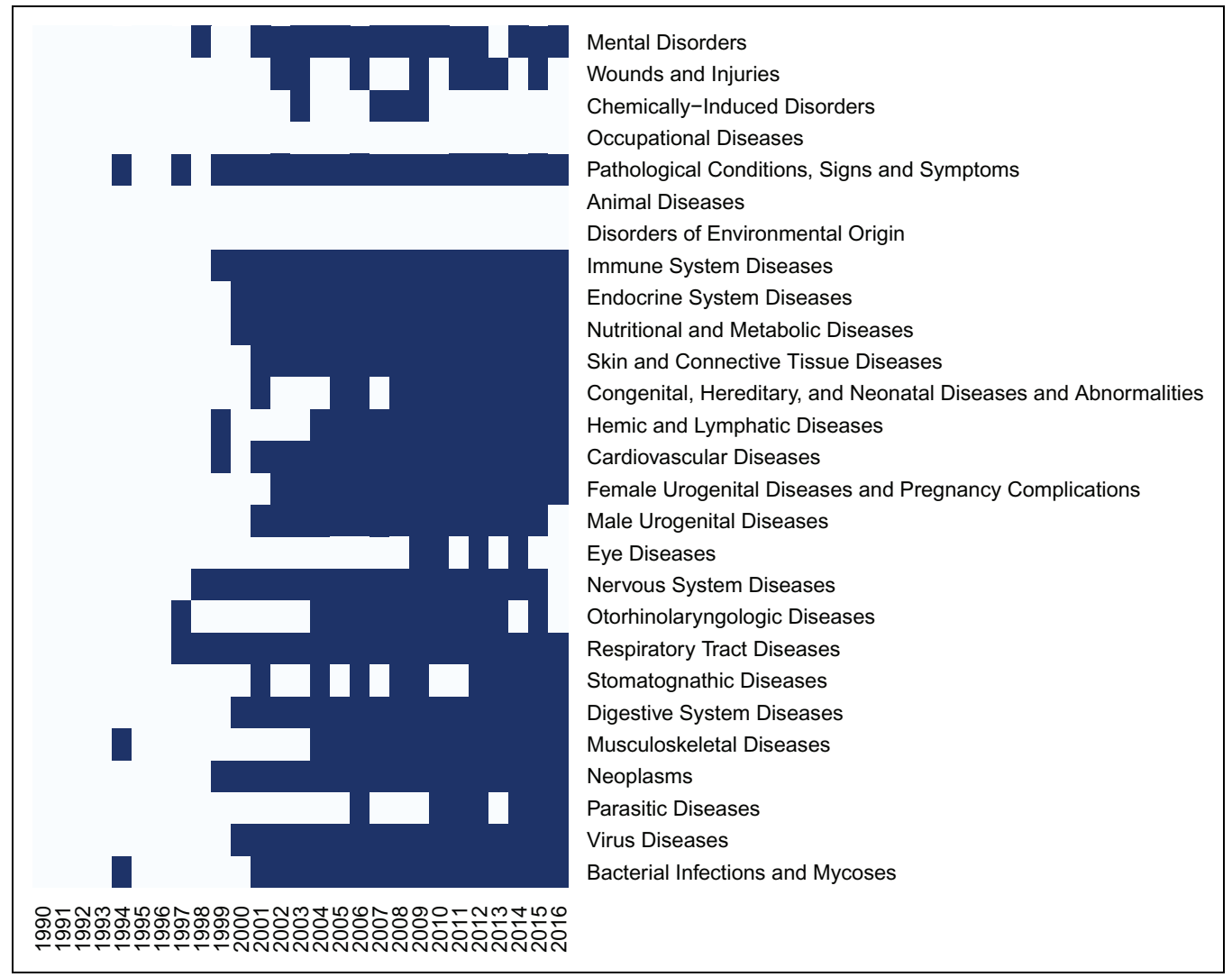

Figure 3. An exemplified therapeutic area map. The horizontal and vertical axes indicate year and 27 therapeutic areas, respectively. A filled cell indicates at least one clinical trial in the therapeutic area in the vertical axis started in the year in the horizontal axis.

Table I. Logistic Regression Analysis.

\begin{tabular}{lcccccc}
\hline Agency Class & $\mathrm{B}$ & SE & $P$ & OR & Lower Cl & Upper Cl \\
\hline Other & 0.105 & 0.053 & $.046^{*}$ & $\mathrm{I} .1 \mathrm{II}$ & $\mathrm{I} .002$ & 1.232 \\
Industry & 0.244 & 0.214 & .254 & $\mathrm{I} .276$ & 0.839 & 1.939 \\
NIH & 0.121 & 0.446 & .786 & $\mathrm{I} .128$ & 0.471 & 2.703 \\
US Fed & 0.667 & 0.568 & .240 & 1.948 & 0.640 & 5.934 \\
Intercept & -5.650 & 0.278 & $<10^{-90 * *}$ & 0.004 & 0.002 & 0.006 \\
\hline
\end{tabular}

Abbreviations: $\mathrm{B}$, regression coefficient; $\mathrm{Cl}$, confidence interval; OR, odds ratio; SE, standard error.

$* P<.05$.

industry collaboration has been expected to be a source of innovation through the $R \& D$ process. ${ }^{23,24}$ In accordance with these trends, the present study aims to explore the effectiveness of alliances on value creation in clinical development using an empirical approach by analyzing the role of industry-academic partnerships in new therapeutic entry. The expansion of usages of marketed drugs is referred to as drug life cycle management or drug repurposing, and the advantages of taking this approach have been extensively discussed in the previous literature. ${ }^{25,26}$ One strategic utility of this approach is oriented to finding new indications through collaborative research activities, ${ }^{27}$ which is expected to contribute to new therapeutic entry by the firm.
This study adopted ClinicalTrials.gov as the primary database of registered clinical trials. This approach has several advantages, including its public availability ${ }^{16}$ and mandatory registration requirement. ${ }^{28}$ Hence, the ClinicalTrials.gov registry has been widely used for investigation of clinical trials. ${ }^{19,29}$ However, the ClinicalTrials.gov registry has some drawbacks. First, the registry may include registration bias. ${ }^{28}$ Pharmaceutical firms were not necessarily proactive in disclosing information at the inception of ClinicalTrials.gov; however, the FDA Amendments Act (FDAAA) enacted in 2007 affected their disclosure policy to register all trials to the database. ${ }^{28}$ Therefore, there is little possibility of being unregistered after 2007, but as pointed out, more attention should be paid to the discontinuity. Second, it does not cover all clinical trials executed in countries other than the United States. In order to address these limitations, data from other registries, such as the European Clinical Trials Database (EudraCT) and the Japan Primary Registries Network (JPRN), are to be further studied in the future.

A key limitation is that although the association between the number of collaboration partners and entry clinical trials is statistically significant, the effect size is relatively small. One possible reason for this small effect size is that the collaborations registered on ClinicalTrials.gov are heterogenous. Analyses with additional information on the collaborations, such as 
type, deal size, and strength, would be valuable for estimating the effect size precisely in specific cases.

The significantly larger number of entries into rare diseases among those trials involving other organizations evokes several advantages for pharmaceutical firms. First, in our observation, 27 of 32 organizations $(84 \%)$ in the other agency class were universities, research institutes, or hospitals. As previously investigated, ${ }^{29}$ the knowledge and expertise held by these types of organizations on unprecedented rare diseases may be effectively transferred to leverage the pharmaceutical firm's capability of drug discovery and development. Second, drug development with an orphan designation can benefit the pharmaceutical firm through various incentives, such as tax credits, exemption from FDA application filing fees, public grants for clinical development, protocol assistance, and marketing exclusivity, that lead to fast regulatory approval. ${ }^{30}$ Third, the approach of using marketed drugs can be used to effectively meet a high level of unmet medical need at a limited cost. These advantages may contribute to mitigating the entry barrier into new therapeutic areas.

\section{Conclusions}

In this open innovation era, alliance in drug development has been increasingly recognized as an important strategy. However, the type of organization that makes the best partner depends on the purpose. In the present study, the comprehensive analyses of clinical trial registry data evaluated the relative impact of each agency class on entry clinical trials and showed that partnership of firms with other organizations, such as universities, hospitals, and research institutes, has a relatively larger effect on therapeutic area entry.

In order to explore possible incentives for alliance for entry, we further investigated the entry clinical trials and revealed that they were likely to target rare diseases and/or leverage marketed drugs. This observation suggests that partnership with other agency class organizations beyond pharmaceutical industry is encouraged to mitigate the entry barrier. We believe that our findings provide a basis for further detailed investigation of these factors for the success of therapeutic area entry.

\section{Acknowledgments}

The authors would thank all the members related to the present research at Tokyo Institute of Technology for critical reading of the manuscript.

\section{Declaration of Conflicting Interests}

Kinya Okada is an employee of Mitsubishi Tanabe Pharma Corporation.

\section{Funding}

The present study was financially and partially supported by the Center of Innovation Program from Japan Science and Technology Agency, JST (COINS).

\section{ORCID iD}

Shintaro Sengoku (D) https://orcid.org/0000-0001-8008-3667

\section{Supplemental Material}

Supplemental material for this article is available online.

\section{References}

1. Kessel M. The problems with today's pharmaceutical businessan outsider's view. Nat Biotechnol. 2011;29(1):27-33.

2. Fernald KDS, Weenen TC, Sibley KJ, Claassen E. Limits of biotechnological innovation. Technol Invest. 2013;4(3):168-178.

3. DiMasi JA, Hansen RW, Grabowski HG. The price of innovation: new estimates of drug development costs. J Health Econ. 2003; 22(2):151-185.

4. Schuhmacher A, Gassmann O, Hinder M. Changing R\&D models in research-based pharmaceutical companies. J Transl Med. 2016; 14(1): 105.

5. Behnke N, Retterath M, van Biesen T. Biopharma strategies: to outperform in pharma, go deep — not broad. In Vivo (Brooklyn). https://invivo.pharmaintelligence.informa.com/IV004544/To-Out perform-In-Pharma-Go-Deep-Not-Broad. Published 2016. Accessed March 19, 2018.

6. Mitchell P. Biogen Idec restructures, sharpens neurology focus. Nat Biotechnol. 2011;29(1):7-8.

7. Davie JM. Biogen and immune disease. Nature. 1999; 402(6763):48.

8. Gilead Sciences to acquire Kite Pharma for $\$ 11.9$ billion. Gilead Sciences, Inc. http://www.gilead.com/news/pressreleases/2017/8/gilead-sciences-to-acquire-kite-pharmafor-119-billion. Published August 28, 2017. Accessed March 19, 2018.

9. Dogramatzis D. Healthcare Biotechnology: A Practical Guide. 1st ed. Boca Raton, FL: CRC Press; 2010.

10. McAfee RP, Hugo M, Mialon HM, Williams MA. What is a barrier to entry? Am Econ Rev. 2004;94(2):461-465.

11. Chen Y, Liu L. Modern methods for delivery of drugs across the blood-brain barrier. Adv Drug Deliv Rev. 2012;64(7): 640-665.

12. Behnke N, Retterath M, Sangster T, Singh A. New paths to value creation in pharma. Bain Br. http://www.bain.com/publications/ articles/new-paths-to-value-creation-in-pharma.aspx. Published 2014. Accessed March 19, 2018.

13. Edwards NV. Pharmaceutical new product development: the increasing role of in-licensing. Anesthesiol Clin. 2008;26(4): 627-636.

14. Sahaf MA. Strategic Marketing: Making Decisions for Strategic Advantage. New Delhi, India: Prentice-Hall India; 2008.

15. Hagedoorn J, Schakenraad J. The effect of strategic technology alliances on company performance. Strategic Manag J. 1994;15: 291-309.

16. Gillen JE, Tse T, Ide NC. Design, implementation and management of a web-based data entry system for ClinicalTrials.gov. Stud Health Technol Inform. 2004;107(pt 2):1466-1470. 
17. Tasneem A, Aberle L, Ananth $\mathrm{H}$, et al. The database for aggregate analysis of Clinicaltrials.gov (AACT) and subsequent regrouping by clinical specialty. PLoS One. 2012;7(3):e33677.

18. Sewell W. Medical subject headings in MEDLARS. Bull Med Libr Assoc. 1964;52:164-170.

19. Califf RM, Zarin DA, Kramer JM, Sherman RE, Aberle LH, Tasneem A. Characteristics of clinical trials registered in ClinicalTrials.gov, 2007-2010. JAMA. 2012;307(17):1838-1847.

20. Step 3: Clinical Research. U.S. Food and Drug Administration Web site. https://www.fda.gov/ForPatients/Approvals/Drugs/ ucm405622.htm. Published January 2018. Accessed April 6, 2018.

21. Orphanet: an online rare disease and orphan drug data base. (C) INSERM 1997. http://www.orphadata.org. Accessed March 20, 2018.

22. Hunter J, Stephens S. Is open innovation the way forward for big pharma? Nat Rev Drug Discov. 2010;9:87-88.

23. Tralau-Stewart CJ, Wyatt CA, Kleyn DE, Ayad A. Drug discovery: new models for industry-academic partnerships. Drug Discov Today. 2009;14(1-2):95-101.
24. Hammonds T. Academic-pharma drug discovery alliances: seeking ways to eliminate the valley of death. Future Med Chem. 2015;7(14):1891-1899.

25. Berger J, Dunn JD, Johnson MM, Karst KR, Shear WC. How drug life-cycle management patent strategies may impact formulary management. Am J Manag Care. 2016;22(16)(suppl): S487-S495.

26. Sachs RE, Ginsburg PB, Goldman DP. Encouraging new uses for old drugs. JAMA. 2017;318(24):2421-2422.

27. Frail DE, Brady M, Escott KJ, et al. Pioneering governmentsponsored drug repositioning collaborations: progress and learning. Nat Rev Drug Discov. 2015;14(12):833-841.

28. Zarin DA, Tse T. Moving toward transparency of clinical trials. Science. 2008;319(5868):1340-1342.

29. Yang H, Lee H. Long-term collaboration network based on ClinicalTrials.gov database in the pharmaceutical industry. Sustainability. 2018;10(2):322.

30. Meekings KN, Williams CSM, Arrowsmith JE. Orphan drug development: an economically viable strategy for biopharma R\&D. Drug Discov Today. 2012;17(13-14):660-664. 